\title{
ERUPTIVE XANTHOMAS AS A PRESENTING FEATURE OF DIABETES MELLITUS
}

\section{Presentation}

A normally fit and well 14year old girl presented to Accident and Emergency with a six week history of widespread itchy, raised and painful rash. It had started over her neck and spread to her torso and limbs. She also complained of polydypsia and polyuria, although no weight loss. She denied any other symptoms including abdominal pain

The family was from the Philippines and there was a strong history of Type 2 diabetes in her mother's family. She has two siblings who are both well.
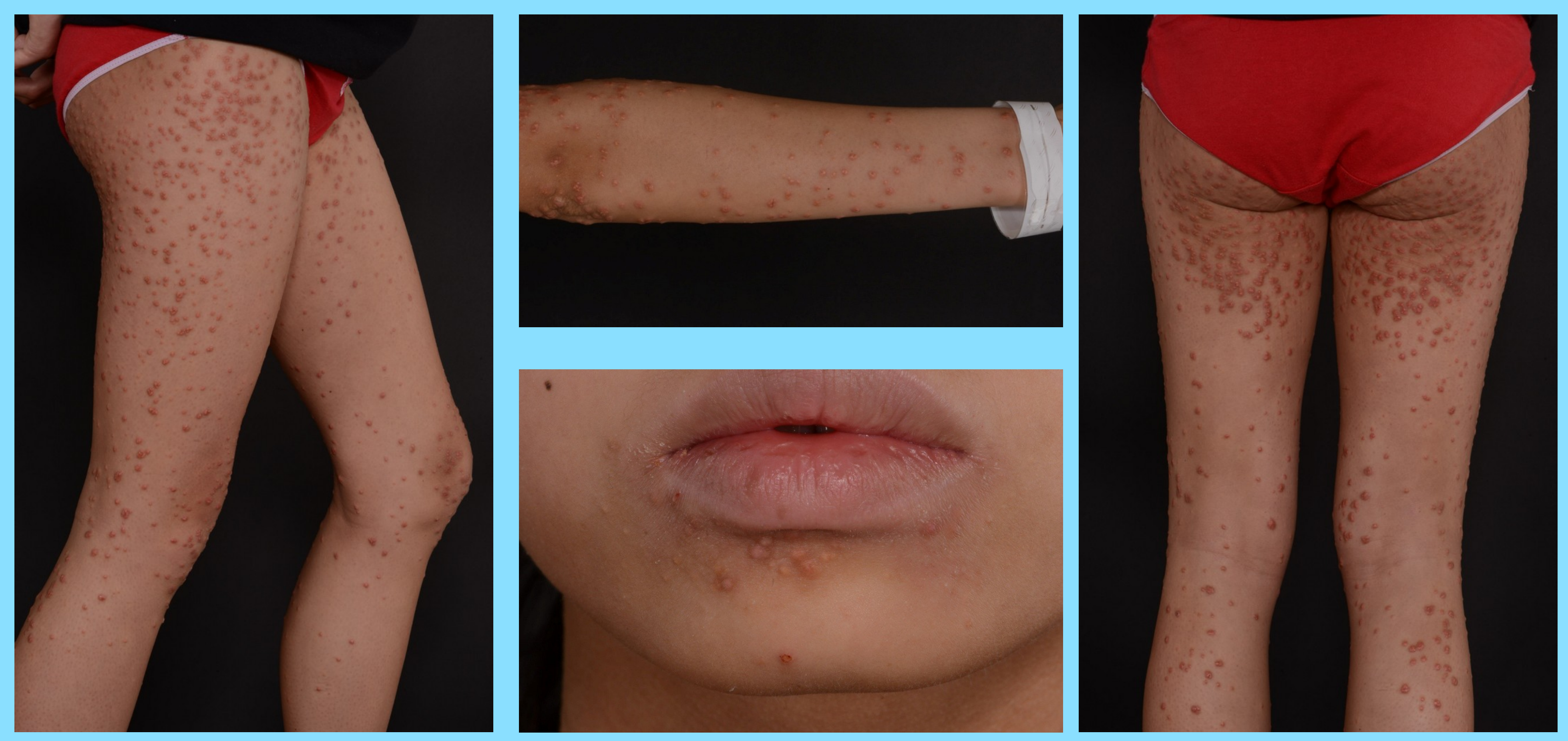

The rash was diagnosed by the dermatology team as eruptive xanthomas.

Eruptive xanthomas are pink papules with creamy centres, often tender and itchy. They predominantly affect the trunk, buttocks and thighs. Histology reveals lipid laden histiocytes and lympho-neutrophilic infiltrate in the dermis. They resolve with improving lipid profile, usually over weeks.

Initial tests supported diagnoses of both hyperlipidaemia and new onset diabetes. ECG was normal.

Blood gas

$\mathrm{pH} 7.36$

$\mathrm{CO}_{2} 4.66$

$\mathrm{BE}-5.3$

Glucose 27

Lactate 0.8

Ketones 5.1

\section{Admission blood Results \\ Cholesterol $34.2 \mathrm{mmol}(<5)$ \\ Triglycerides $189 \mathrm{mmol}(1.7)$ \\ HDL $0.8 \mathrm{mmol}(>1.2)$ \\ Amylase 85}

HbA1c $17.7 \%$

$\mathrm{TSH} 2.4$, free $\mathrm{T}_{4} 19$ C-peptide 0.35 Urate 216 (normall)

She had previously been for a routine eye check where she was told her eyes looked 'cloudy'. On ophthalmological examination she was diagnosed with lipaemia retinalis.

ipaemia retinalis is a rare presentation occurring at triglyceride concentrations over $45 \mathrm{mmol} / \mathrm{l}$. The retinal vessels have a milky appearance, as seen below. This is due to visualisation of high levels of chylomicrons in the vessels and resolves as these lower.
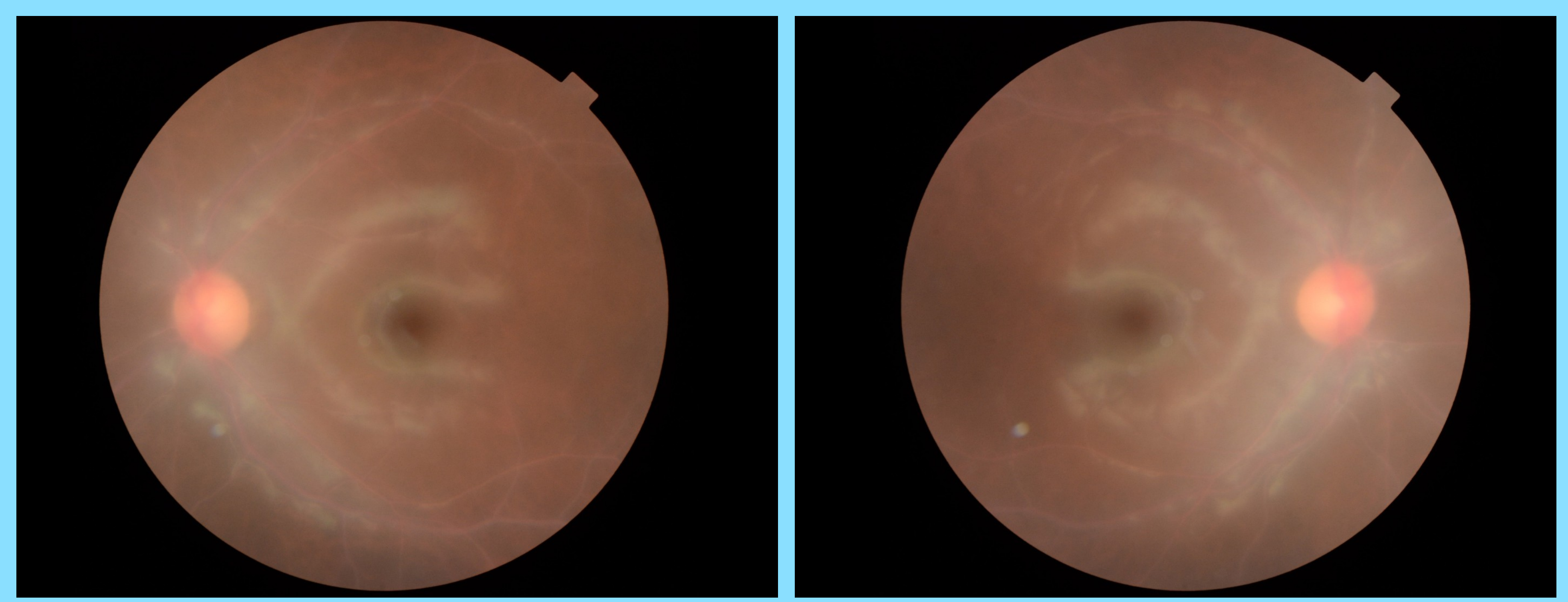

Abdominal ultrasound scan showed xanthogranulomatous infiltration of both kidneys. This is demonstrated by distinctive bands of hyper-echogenicity around the pyramids seen in her pictures below.
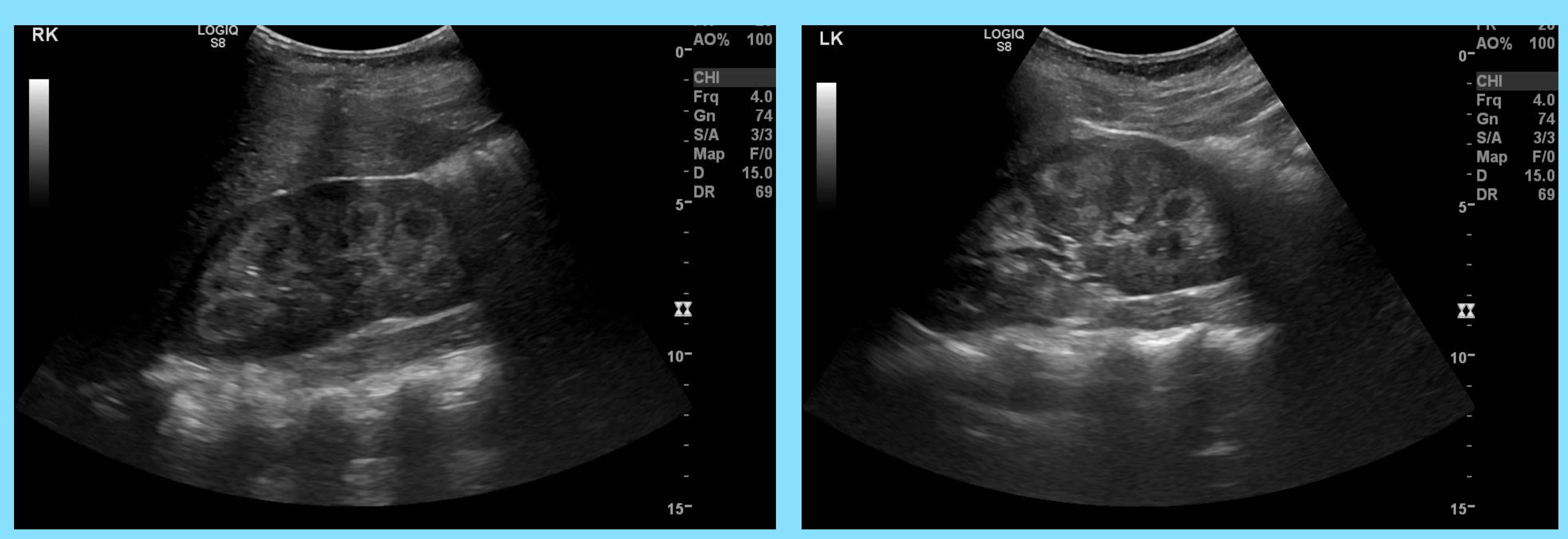

Diabetic antibodies

Autoantibody screen negative Anti-GAD $<5 \mathrm{U} / \mathrm{ml}$

Anti-pancreatic islet cell Negative
Lipid electrophoresis - Type 5 hyperlipidaemia

Genetic analysis - no pathogenic mutations typical of Type 1 or 5 familial hyperlipidaemia

\section{Management}

Total 10 day admission for education and to achieve safe lipid profile

Diabetes:

Glucose control was initially erratic

Established on Levemir and Novorapid basal bolus regime

Discharged on 0.8 units $/ \mathrm{kg} /$ day insulin

Hyperlipidaemia (discussed with Consultants of biochemistry and metabolic medicine): Commenced on Fibrate

Extremely low fat diet $(10-15 \%$ of calorie intake $)<20$ g/day
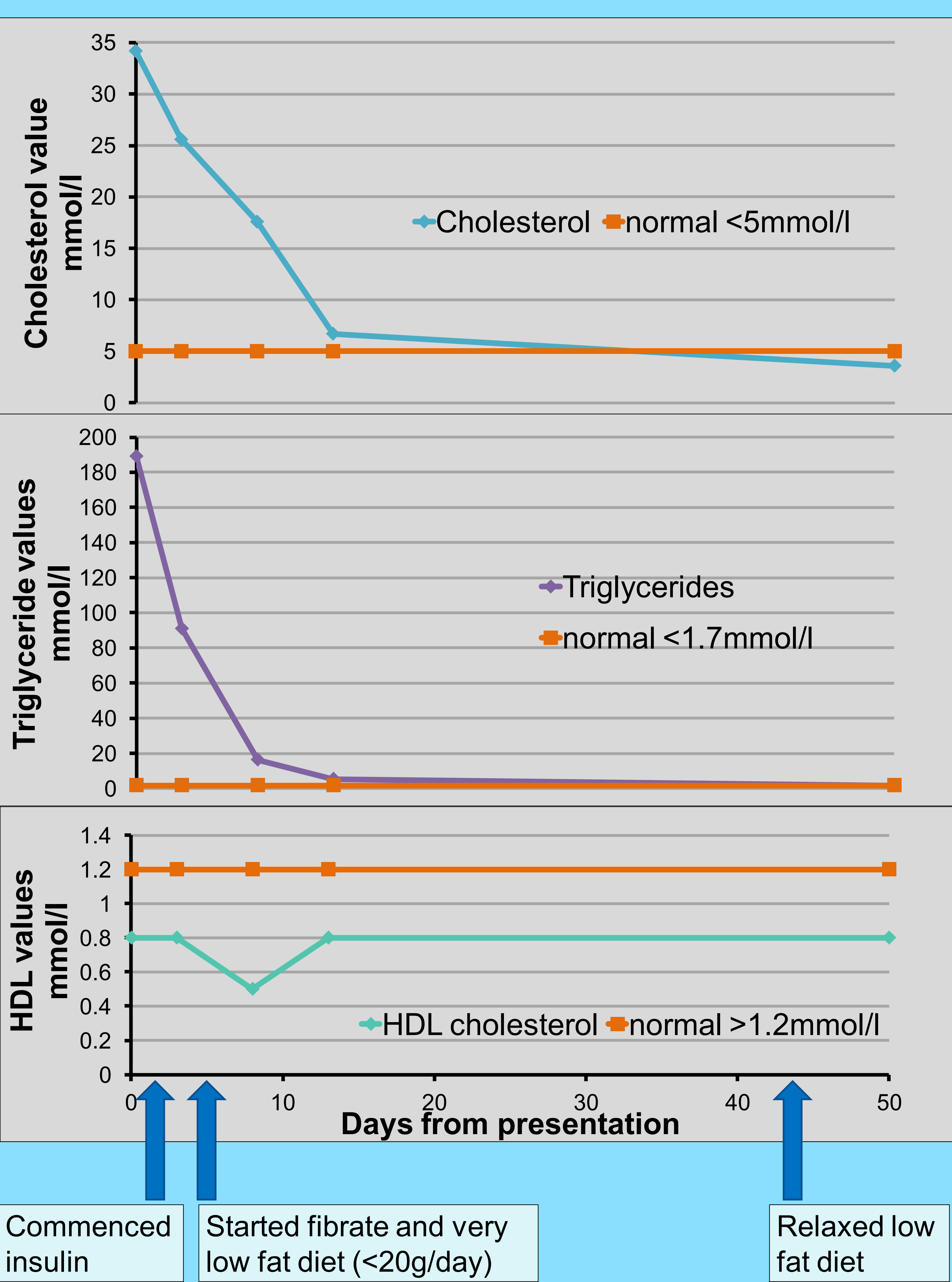

insulin $\quad$ low fat diet $(<20 \mathrm{~g} /$ day $)$

fat diet

Results at 4 months

Cholesterol $3.9 \mathrm{mmol}(<5)$

Triglycerides $1.8 \mathrm{mmol}(1.7)$

$\mathrm{HDL} 1.1 \mathrm{mmol}(>1.2)$

$\mathrm{HbA} 1 \mathrm{c} 55 \mathrm{mmol} / \mathrm{mol}(7.2 \%)$

\section{Conclusion}

This case illustrates a young person whose initial presentation with eruptive xanthomas led to a diagnosis of new onset diabetes, complicated by severe hyperlipidaemia.

Hyperlipidaemia in children presenting in severe Diabetic Ketoacidosis (DKA) is a known, but rarely reported, phenomenon. The triglyceride levels in this case are higher than any other we could find There is only one other report of a child presenting similarly with new onset diabetes and eruptive xanthomas that we could find ${ }^{1}$.

In these cases care needs to be taken to evaluate the risk of pancreatitis, and there should be an awareness that lipaemia may make serum electrolyte measurement inaccurate - affecting DKA management ${ }^{2}$.

Previous proposals to explain this phenomenon include genetic abnormalities of lipoprotein lipase $^{3}$, or a transient decrease in lipoprotein lipase activity secondary to insulin deficiency ${ }^{2,4}$

Management strategies are not standard and mostly derive from the treatment of hyperlipidaemic pancreatitis. These include plasmapheresis ${ }^{2,5}$, fat-free total parenteral nutrition, insulin, heparin and antihyperlipidaemic drugs ${ }^{5}$.

However, in many reports lipid profile improved with standard management of DKA alone.

There is no current work that we could find which attempts to explain the differences in severity of dyslipidaemia in children with new onset type 1 diabetes. 